\title{
Efficient Algorithms for the Evaluation of the Eigenvalues of (Block) Banded Toeplitz Matrices*
}

\author{
By D. Bini and V. Pan
}

\begin{abstract}
Let $A$ be an $n \times n$ banded block Toeplitz matrix of bandwidth $k$ with $m \times m$ blocks having entries in a field $\mathbf{F}$. We present algorithms for computing $p(\lambda)=$ $\operatorname{det}(A-\lambda I)$ as well as the ratio $p(\lambda) / p^{\prime}(\lambda)$, where $p^{\prime}(\lambda)$ is the first derivative of $p(\lambda)$ with respect to $\lambda$, in roughly $(3 / 2) k^{2} \log n+O\left(k^{3}\right)$ block multiplications. If the field F supports FFT, then the cost is reduced to $O\left(\left(m^{2} k \log k+m^{3} k\right) \log n+k^{3} m^{3}\right)$ scalar multiplications. The algorithms generalize an algorithm given by $\mathrm{W}$. Trench for computing $p(\lambda)$ in the case $m=1$ in roughly $k \log n+O\left(k^{3}\right)$ multiplications and rely on powering a companion matrix associated with the linear recurrence relation representing the original problem.
\end{abstract}

1. Introduction. Toeplitz matrices, either with scalar entries or with blocks, arise in a variety of problems of Applied Mathematics; a wide literature deals with the problem of their inversion. Recently, some interest has been focused on the eigenvalue problem for banded Toeplitz matrices. In [1] and [2], spectral properties are expressed in terms of a suitable polynomial associated with a banded Toeplitz matrix $A$, and parallel algorithms have been devised for the computation of the characteristic polynomial $p(\lambda)=\operatorname{det}(A-\lambda I)$ at a point $\lambda$ as well as of the ratio $p(\lambda) / p^{\prime}(\lambda)$, where $p^{\prime}(\lambda)$ is the first derivative of $p(\lambda)$.

More recently, an algorithm for the computation of $p(\lambda)$ has been proposed by Trench [8]; its cost is roughly $k \log _{2} n+O\left(k^{3}\right)$ multiplications, where $n$ is the dimension of the matrix and $k$ is its bandwidth. That algorithm works over any algebraically closed field (even though it was originally presented over the complex field), and it is assumed that the zeros of a suitable $k$ th degree polynomial, associated with the banded Toeplitz matrix, have been precomputed together with their multiplicities.

In this paper we consider the eigenvalue problem for banded block Toeplitz matrices and present two new algorithms for the computation of $p(\lambda)$. The first algorithm, which is obtained dealing with the problem in terms of a linear difference ma-

Received October 17, 1986; revised August 28, 1987.

1980 Mathematics Subject Classification (1985 Revision). Primary 65F15.

Key words and phrases. Banded Toeplitz matrices, block matrices, eigenvalues, computational complexity, matrix difference equation, cyclic reduction.

${ }^{*}$ The first author was supported by NSF Grant DCR 8507573 and by M.P.I. $40 \%$ funds, and the second author was supported by NSF Grant DCR 8507573.

** The abstract appeared in Abstracts of papers presented to the Amer. Math. Soc., v. 8, no. 1, 1987, p. 145 (87T-68-14), and in Abstracts of ICIAM'87, INRIA, France 1987. The main results of this paper have been presented at the Oberwolfach conference on Complexity, November 1986. 
trix equation by using block companion matrices, requires roughly $(3 / 2) k^{2} \log _{2} n+$ $O\left(k^{3}\right)$ block multiplications, works over any field and does not require any precomputation of approximations to polynomial zeros. Moreover, if the ground field supports FFT, then we may reduce the cost to $O\left(\left(m^{2} k \log k+m^{3} k\right) \log n+k^{3} m^{3}\right)$ arithmetic operations by using fast polynomial arithmetic, where $m$ is the dimension of the blocks. In the case of a tridiagonal block Toeplitz matrix the cost is given by $5 \log _{2} n-3$ block multiplications (observe that almost any banded block Toeplitz matrix can be considered as a tridiagonal block Toeplitz matrix having blocks of a suitable size).

The second algorithm, based on a cyclic reduction method, applies to tridiagonal block Toeplitz matrices and computes $p(\lambda)$ in roughly $7 \log _{2} n$ block multiplications, requiring the nonsingularity of certain auxiliary matrices which must be inverted.

Those algorithms approximate to the eigenvalues of the matrix $A$ as zeros of its characteristic polynomial $p(\lambda)$. They can be combined with any rootfinding method which requires only values of $p(\lambda)$, but not its coefficients.

Both our algorithms can be easily extended to the computation of $p(\lambda) / p^{\prime}(\lambda)$ at roughly double the cost, so that Newton's method can be efficiently applied to the equation $p(\lambda)=0$ for approximating the eigenvalues of the matrix $A$.

The algorithms can be easily adapted to the computation of $p(\lambda)=\operatorname{det}(A-\lambda B)$ as well as of $p(\lambda) / p^{\prime}(\lambda)$, where $A$ and $B$ are banded block Toeplitz matrices, so that the generalized eigenvalue problem can also be treated.

The paper is organized as follows. In Section 2 we describe the algorithm for computing $p(\lambda)$ by powering a companion matrix, in the scalar case. In Section 3 we consider the computation of the ratio $p(\lambda) / p^{\prime}(\lambda)$; in Section 4 we extend the computations to the case of block matrices. In Section 5 the degenerate case is considered; in Section 6 we describe the algorithms based on cyclic reduction.

2. Evaluation of the Determinant of a Banded Toeplitz Matrix. Let $\mathbf{F}$ be a field and $A-\lambda I$ be an $(r+s+1)$-diagonal $n \times n$ Toeplitz matrix,

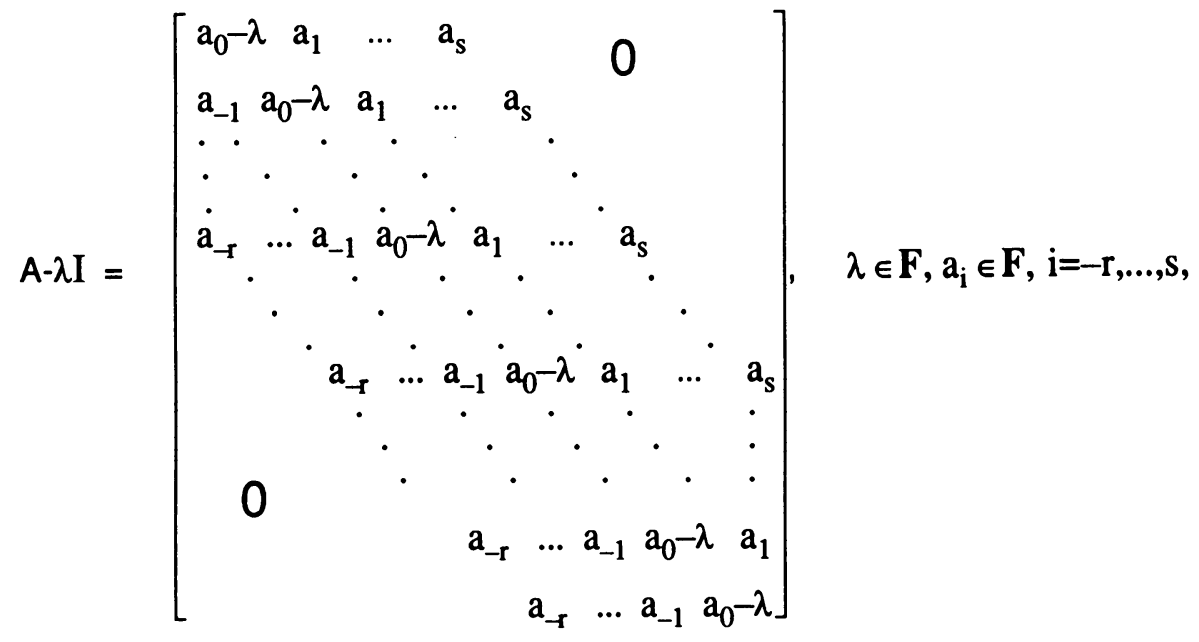


where $a_{-r}, \ldots, a_{s} \in \mathbf{F}, a_{-r}, a_{s} \neq 0$. We consider the problem of computing $p(\lambda)=$ $\operatorname{det}(A-\lambda I)$, that is, the value that the characteristic polynomial of $A$ takes on at a point $\lambda$.

The assumption that $(\lambda, \mathbf{x})$ is an eigenvalue-eigenvector pair for $A$, that is,

$$
(A-\lambda I) \mathbf{x}=\mathbf{0}, \quad \mathbf{x}=\left(x_{i}\right) \neq \mathbf{0},
$$

is equivalent to the following linear system of equations

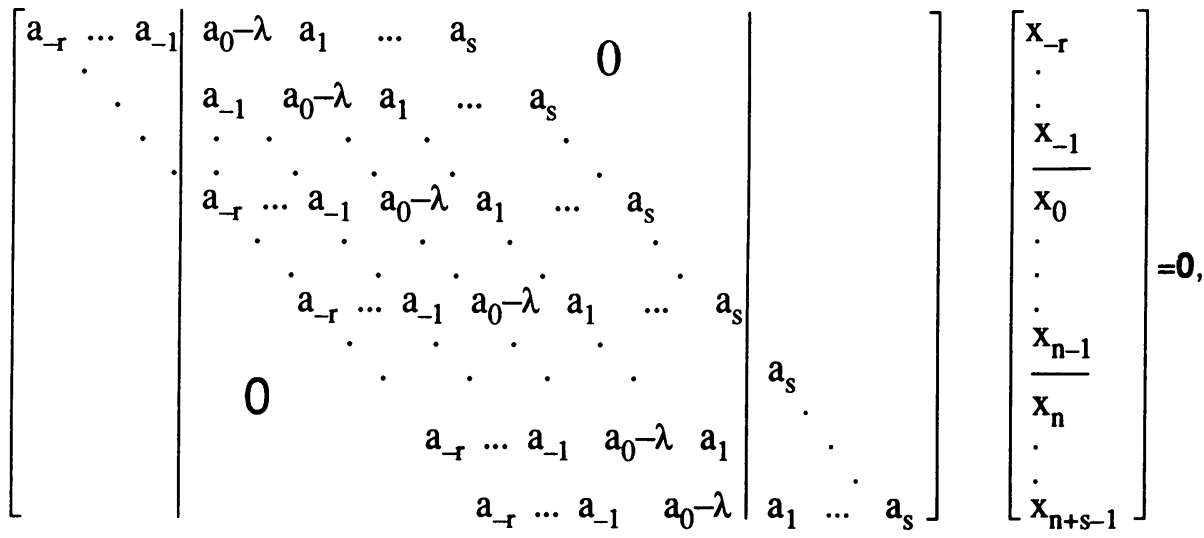

$$
\begin{aligned}
& x_{-r}, \ldots, x_{-1}=0 \text {, } \\
& x_{n}, \ldots, x_{n+s-1}=0 \text {. }
\end{aligned}
$$

that is, to the following constant-coefficient homogeneous difference equations,

$$
\begin{array}{r}
a_{s} x_{i+k}+a_{s-1} x_{i+k-1}+\cdots+\left(a_{0}-\lambda\right) x_{r+i}+\cdots+a_{-r} x_{i}=0, \\
i=-r,-r+1, \ldots, n-r-1, a_{s} \neq 0, k=r+s,
\end{array}
$$

with the "boundary" conditions

$$
\begin{array}{ll}
x_{-r}, \ldots, x_{-1}=0 & \text { (initial conditions) } \\
x_{n}, \ldots, x_{n+s-1}=0 & \text { (terminal conditions) }
\end{array}
$$

This fact was used by Trench [8] to give an explicit expression for $\operatorname{det}(A-\lambda I)$ in the case where $\mathbf{F}$ is the complex field. The same result holds in the more general case where the field $\mathbf{F}$ has an algebraic closure $\mathbf{G}$. The main idea of [8] is to express the general solution of (2.2) in terms of the zeros $z_{1}, \ldots, z_{k} \in \mathbf{G}$ of the associated polynomial

$$
\begin{aligned}
\varphi(z)=z^{k} & +\sum_{j=0}^{k-1} \gamma_{j} z^{j}, \\
\gamma_{j} & =a_{s}^{-1} a_{j-2}, j=0, \ldots, k-1, j \neq r, \gamma_{r}=a_{s}^{-1}\left(a_{0}-\lambda\right) .
\end{aligned}
$$

If, for simplicity, we suppose that $z_{i} \neq z_{j}, i \neq j$, then the general solution of the difference equation (2.2) is given by [6, Section 7.2.9]

$$
x_{i}=\sum_{j=1}^{k} \alpha_{j} z_{j}^{i+r}, \quad i=-r,-r+1, \ldots, n+s-1, \alpha_{j} \in \mathbf{G}, j=1, \ldots, k .
$$


Moreover, $\lambda$ is an eigenvalue of $A$ if and only if not all of the $x_{i}$ are zero, or, equivalently, if and only if not all of the $\alpha_{j}$ are zero, since the row vector formed by $x_{i}$ equals the product of the row vector $\alpha_{j}$ times a Vandermonde matrix. Imposing the boundary conditions $(2.3)$ yields

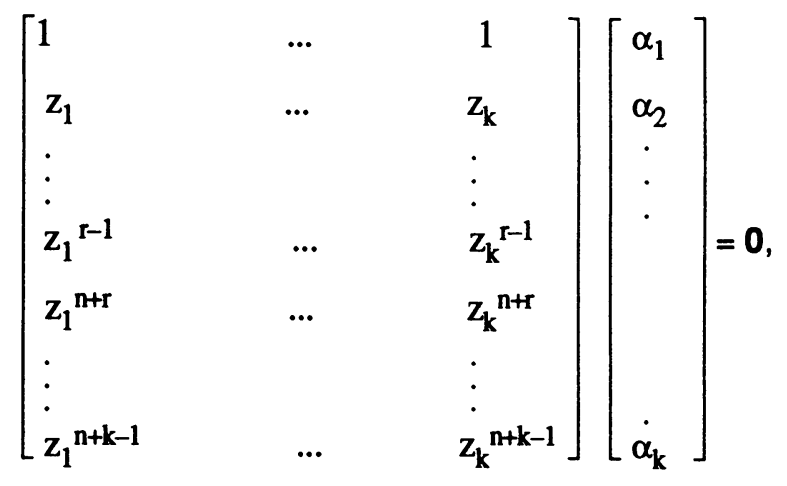

that is, $\lambda$ is an eigenvalue of $A$ if and only if $\operatorname{det} V_{n}=0$, where $V_{n}$ is the $k \times k$ matrix of this last system. Trench shows that

$$
\operatorname{det}(A-\lambda I)=(-1)^{n s} a_{s}^{n} \operatorname{det} V_{n} / \operatorname{det} V_{0}
$$

where $V_{0}=\left(z_{j}^{i-1}\right)$ is the $k \times k$ Vandermonde matrix associated with the zeros $z_{j}$; he also extends this last equation to the case where the condition $z_{i} \neq z_{j}$, $i \neq j$, is not satisfied. Observe that if the zeros $z_{j}$ are known, or if they can be easily approximated, then it is possible to compute $p(\lambda)$ in roughly $k \log _{2} n+O\left(k^{3}\right)$ multiplications. In this way, to approximate the eigenvalues of $A$ as the zeros of $p(\lambda)$, it is possible to apply the secant method or any rootfinding method to the polynomial equation $p(\lambda)=0$, which uses only the values of $p(\lambda)$ at $\lambda$.

Now we present a different approach, which yields an algorithm for the evaluation of $p(\lambda)$ over an arbitrary field of constants F. That algorithm does not require to precompute approximations to the zeros of an auxiliary polynomial; furthermore, it enables us to extend the result to the case of block Toeplitz matrices and to compute the ratio $p(\lambda) / p^{\prime}(\lambda)$, which in turn allows us to apply Newton's method to the polynomial equation $p(\lambda)=0$ in order to evaluate the eigenvalues of the matrix $A$.

First we observe that the matrix vector equation (2.1) is equivalent to the linear recurrence (2.2) with the boundary condition (2.3) and can be rewritten as follows:

$$
\left[\begin{array}{c}
x_{i} \\
\vdots \\
x_{i+k-1}
\end{array}\right]=F^{r+i}\left[\begin{array}{c}
x_{-r} \\
\vdots \\
x_{s-1}
\end{array}\right], \quad i=-r,-r+1, \ldots, n-r
$$


Here, $F$ is the $k \times k$ companion (Frobenius) matrix,

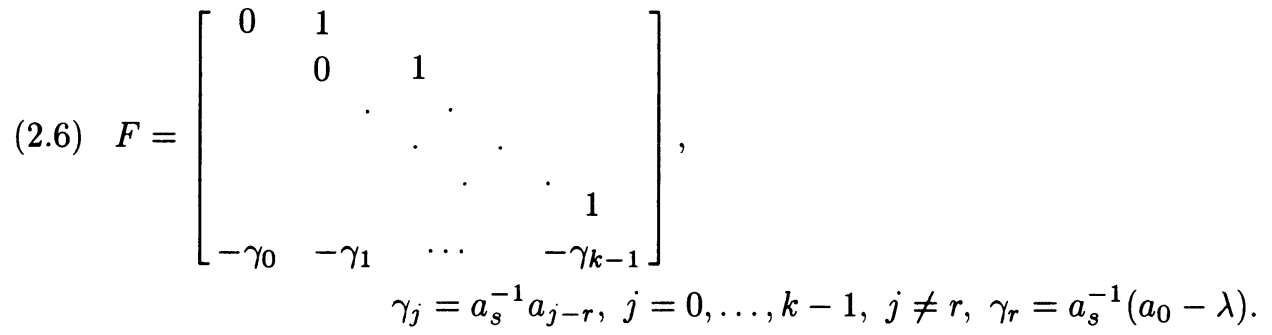

Setting $\mathbf{y}=\left(x_{-r}, x_{-r+1}, \ldots, x_{s-1}\right)^{T} \in \mathbf{F}^{k}$, we can rewrite (2.3) as follows,

$$
\begin{array}{ll}
{\left[I_{r} \mid 0\right] \mathbf{y}=\mathbf{0}} & \text { (initial conditions), } \\
{\left[0 \mid I_{s}\right] F^{n} \mathbf{y}=\mathbf{0}} & \text { (terminal conditions), }
\end{array}
$$

where $F$ is defined by (2.6). Here and hereafter, $I_{m}$ denotes the $m \times m$ identity matrix, $\mathbf{v}^{T}$ denotes the transpose of $\mathbf{v}$. The second equation follows from (2.3) and (2.5) where $i=n-r$. Setting

$$
[V \mid U]=\left[0 \mid I_{s}\right] F^{n}
$$

we deduce that $(2.1)$ is equivalent to

$$
\left[\begin{array}{cc}
I_{r} & 0 \\
V & U
\end{array}\right] \mathbf{y}=\mathbf{0}, \mathbf{y} \neq \mathbf{0}
$$

that is, to the equation $\operatorname{det} U=0$, so the following relation holds:

$$
p(\lambda)=0 \text { if and only if } \operatorname{det} U=0 .
$$

From this, we deduce the following result.

PROPOSITION 2.1. Let $U$ be the right lower $s \times s$ submatrix of $F^{n}$; then for the characteristic polynomial $p(\lambda)$ of $A$ we have

$$
p(\lambda)=(-1)^{n s} a_{s}^{n} \operatorname{det} U .
$$

ProOF. First observe that $(-1)^{n} p(\lambda)$ is a monic polynomial of degree $n$; we show that $(-1)^{n(s+1)} a_{s}^{n} \operatorname{det} U$ is also a monic polynomial of degree $n$, that is, the term of $\operatorname{det} U$ having the highest degree in $\lambda$ is $(-1)^{n(s+1)} \lambda^{n} a_{s}^{-n}$. For this purpose, let $U_{q}, q=1,2, \ldots, n$, denote the $s \times s$ right lower submatrix of $F^{q}$ in which the entries not contributing to the term of the highest degree in $\lambda$ are set to zero. Then, if $g$ and $e$ are natural numbers such that $q=g s+e, e<s$, we have that

$$
U_{q}=\left[\begin{array}{cc}
0 & \left(\lambda a_{s}^{-1}\right)^{g} I_{s-e} \\
\left(\lambda a_{s}^{-1}\right)^{g+1} I_{e} & 0
\end{array}\right] .
$$

The last equation is trivial for $q=1$ and is easily verified by induction on $q$, using the relation

$$
F^{q+1}=F F^{q}=H F^{q}+\mathbf{i}_{k} \mathbf{g}^{T} F^{q},
$$

where $H=\left(h_{i, j}\right), h_{i, i+1}=1, h_{i, j}=0$ otherwise, $\mathbf{i}_{k}$ is the last column of $I_{k}$ and $-\mathbf{g}^{T}=\left(\gamma_{0}, \ldots, \gamma_{k-1}\right)$. (Indeed, $H F^{q}$ is obtained by shifting upwards the rows of $F^{q}$, and the highest-degree entry of the matrix $\mathbf{i}_{k} \mathbf{g}^{T} F^{q}$ is $\left(\lambda a_{s}^{-1}\right)^{g+1}$, in the last 
row and in the $(r+e+1)$ st column. Therefore, the term of highest degree in $\operatorname{det} U$ is given by

$$
\operatorname{det} U_{n}=(-1)^{n(s+1)} \lambda^{n} a_{s}^{-n} .
$$

Now, (2.8) follows if $p(\lambda)$ has pairwise distinct zeros, since

$$
(-1)^{n} p(\lambda) \text { and }(-1)^{n(s+1)} a_{s}^{n} \operatorname{det} U
$$

are both monic polynomials and have $n$ distinct common zeros. The result is immediately extended via a continuity argument to the case where $p(\lambda)$ has multiple zeros.

We will also need the following

PROPOSITION 2.2. If $\psi(z)=z^{n} \bmod \varphi(z)$, then $\psi(F)=F^{n}$.

Proposition 2.2 immediately follows from the well-known fact $[6$, p. 325] that the polynomial (2.4) is the minimal polynomial of the Frobenius matrix $F$, that is, $\varphi(F)=0$.

Now we are ready to present the following algorithm for the evaluation of $p(\lambda)$, which, for simplicity, will be described under the assumption that $n=2^{h}, h$ integer.

Stage 1. Compute the coefficients of the polynomial

$$
\psi(z)=\sum_{i=0}^{k-1} \alpha_{i} z^{i}=z^{n} \bmod \varphi(z),
$$

by using the repeated squaring technique:

$$
\psi_{0}=z, \quad \psi_{i+1}=\psi_{i}^{2} \bmod \varphi(z), \quad i=0, \ldots, h-1, h=\log _{2} n, \psi_{h}=\psi
$$

Stage 2. Compute the matrix

$$
U=\left[0 \mid I_{s}\right] F^{n}\left[\begin{array}{c}
0 \\
I_{s}
\end{array}\right]=\left[0 \mid I_{s}\right] \psi(F)\left[\begin{array}{c}
0 \\
I_{s}
\end{array}\right]
$$

by means of Horner's rule,

$$
U=\alpha_{0} L+\left(\alpha_{1} L+\left(\cdots+\left(\alpha_{k-2} L+\alpha_{k-1} L F\right) F\right) \cdots\right) F\left[\begin{array}{c}
0 \\
I_{s}
\end{array}\right]
$$

where $L=\left[0 \mid I_{s}\right]$ is a $s \times k$ matrix, according to the following steps: successively compute

$$
\begin{aligned}
Y_{1} & =\alpha_{k-1} L, \\
Y_{i} & =\left(\alpha_{k-i} L+Y_{i-1} F\right), \quad i=2, \ldots, k, \\
U & =Y_{k}\left[\begin{array}{c}
0 \\
I_{s}
\end{array}\right] .
\end{aligned}
$$

Stage 3. Compute $(-1)^{n s} a_{s}^{n} \operatorname{det} U$.

If we compute over the field of complex numbers or over any other field that supports FFT, we may use fast polynomial arithmetic in order to multiply and divide a pair of $k$ th degree polynomials in $O(k \log k)$ arithmetic operations (see [3]); in that case, the cost of the algorithm is $O\left(k \log k \log (n / k)+k^{3}\right)$ multiplications. On the other hand, we may apply customary polynomial arithmetic with the cost of roughly $(3 / 2) k^{2} \log (n / k)+O\left(k^{3}\right)$ multiplications. 
For comparison, the method given in [8] evaluates $p(\lambda)$ as follows.

Stage 1. Compute all the zeros $z_{1}, \ldots, z_{k}$ of $\varphi(z)$ together with their multiplicities.

Stage 2. Compute a $k \times k$ Vandermonde-like matrix $V_{n}$ depending on the multiplicities of the zeros. If $z_{i} \neq z_{j}$ for $i \neq j$, this matrix has elements $z_{j}^{i}$ for $i=0, \ldots, r-1$ and $z_{j}^{n+i}$ for $i=r, \ldots, r+s-1$.

Stage 3. Compute $(-1)^{n s} a_{s}^{n} \operatorname{det} V_{n} / \operatorname{det} V_{0}$.

The cost of this method is given by $k \log _{2} n+O\left(k^{3}\right)$ multiplications, not counting the cost of Stage 1, which can be rather large, particularly in the case of clustered zeros (estimated in [5]). This is less than the cost of our algorithm by a factor of $\log k$, if we compute over fields supporting FFT, and by a factor of $k$ otherwise.

The new approach yields a "rational" algorithm, that is, an algorithm which does not compute any approximations to polynomial zeros and thus, in exact arithmetic, delivers the exact result in a finite number of arithmetical operations. Moreover, this method can be slightly modified in order to deal with block matrices and to compute the ratio $p(\lambda) / p^{\prime}(\lambda)$, where $p^{\prime}(\lambda)$ is the first derivative of $p(\lambda)$ with respect to $\lambda$. These two generalizations are the subject of the next three sections.

3. Evaluation of the Ratio $p(\lambda) / p^{\prime}(\lambda)$. In order to evaluate the eigenvalues of $A$, applying Newton's method to the polynomial equation $p(\lambda)=0$, we must compute the quotient $p(\lambda) / p^{\prime}(\lambda)$. From (2.5) and (2.8) we deduce the equation $p^{\prime}(\lambda) / p(\lambda)=(\operatorname{det} U)^{\prime} /(\operatorname{det} U)$. Recall that

$$
(\operatorname{det} U)^{\prime}=\sum_{i, j} \frac{\partial \operatorname{det} U}{\partial u_{i j}} \frac{d u_{i j}}{d \lambda}=\operatorname{trace}\left((\operatorname{Adj} U) U^{\prime}\right)
$$

where $U^{\prime}=\left(u_{i j}^{\prime}\right), u_{i j}^{\prime}=d u_{i j} / d \lambda$, and $\operatorname{Adj} U=U^{-1} \operatorname{det} U$ is the adjugate matrix of $U$. Consequently,

$$
(\operatorname{det} U) /(\operatorname{det} U)^{\prime}=1 / \operatorname{trace}\left(U^{-1} U^{\prime}\right) .
$$

Thus, we essentially reduced the problem to computing $U^{-1}$ and $U^{\prime}$, the $s \times s$ lower right submatrices of $F^{n}$ and $\left(F^{n}\right)^{\prime}$. Since $F^{n}=\psi(F)$, where

$$
\psi(z)=\sum_{i=0}^{k-1} \alpha_{i} z^{i}=z^{n} \bmod \varphi(z)
$$

and $\varphi(z)=\varphi(z, \lambda)$ is defined by $(2.4)$, we have the relation

$$
\left(F^{n}\right)^{\prime}=\sum_{i=0}^{k-1} \alpha_{i}^{\prime} F^{i}+\sum_{i=0}^{k-1} \alpha_{i}\left(F^{i}\right)^{\prime}
$$

so that

$$
U^{\prime}=G+H, \quad G=L \sum_{i=0}^{k-1} \alpha_{i}^{\prime} F^{i}\left[\begin{array}{c}
0 \\
I_{s}
\end{array}\right], \quad H=L \sum_{i=0}^{k-1} \alpha_{i}\left(F^{i}\right)^{\prime}\left[\begin{array}{c}
0 \\
I_{s}
\end{array}\right]
$$

where $L=\left[0 \mid I_{s}\right]$. 
Next we will show how to compute simultaneously $H$ and $U$. Observe that

$$
\begin{aligned}
\sum_{i=0}^{k-1} \alpha_{i}\left(F^{i}\right)^{\prime} & =\sum_{i=1}^{k-1} \alpha_{i}\left(F^{i-1} F\right)^{\prime}=\sum_{i=1}^{k-1} \alpha_{i}\left(F^{i-1} F^{\prime}+\left(F^{i-1}\right)^{\prime} F\right) \\
& =\sum_{i=1}^{k-1} \alpha_{i} F^{i-1} F^{\prime}+\sum_{i=1}^{k-1} \alpha_{i}\left(F^{i-1}\right)^{\prime} F=\sum_{i=1}^{k-1} \alpha_{i} F^{i-1} F^{\prime}+\sum_{i=0}^{k-2} \alpha_{i+1}\left(F^{i}\right)^{\prime} F
\end{aligned}
$$

Setting $Y_{i}=L \sum_{j=1}^{i} \alpha_{k-i+j-1} F^{j-1}$ and $Z_{i}=L \sum_{j=0}^{i-1} \alpha_{k-i+j}\left(F^{j}\right)^{\prime}$, we get the relations

$$
\begin{aligned}
& Y_{1}=L \alpha_{k-1}, \quad Z_{1}=L \alpha_{k-1}, \\
& Y_{i}=Y_{i-1} F+L \alpha_{k-i}, \quad Z_{i}=Y_{i-1} F^{\prime}+Z_{i-1} F, \quad i=2,3, \ldots, k, \\
& H=Z_{k}\left[\begin{array}{c}
0 \\
I_{s}
\end{array}\right], \quad U=Y_{k}\left[\begin{array}{c}
0 \\
I_{s}
\end{array}\right] .
\end{aligned}
$$

It remains to compute the first term in the right-hand side of (3.2). Rewrite (2.9) as follows,

$$
\begin{aligned}
& \psi_{0}=z \\
& \psi_{i}^{2}=Q_{i} \varphi+\psi_{i+1}, \quad i=0, \ldots, h-1, h=\log _{2} n \\
& \psi_{h}=\psi(z)
\end{aligned}
$$

where $\varphi=\varphi(z)$. Taking the derivatives with respect to $\lambda$, we obtain that

$$
\begin{aligned}
& \psi_{0}^{\prime}=0, \\
& 2 \psi_{i} \psi_{i}^{\prime}=Q_{i}^{\prime} \varphi+Q_{i} \varphi^{\prime}+\psi_{i+1}^{\prime}, \quad i=0, \ldots, h-1, \\
& \psi_{h}^{\prime}=\psi^{\prime} .
\end{aligned}
$$

Since $\varphi^{\prime}=-z^{r}$, we have that $2 \psi_{i} \psi_{i}^{\prime}+Q_{i} z^{r}=Q_{i}^{\prime} \varphi+\psi_{i+1}^{\prime}$ (that is, $\psi_{i+1}^{\prime}=$ $\left.2 \psi_{i} \psi_{i}^{\prime}+Q_{i} x^{r} \bmod \varphi\right)$. Therefore, the derivative with respect to $\lambda$ of the polynomial $\psi(z)$ can be evaluated together with $\psi(z)$ using the following equations:

$$
\left.\begin{array}{l}
\psi_{0}=z, \quad \psi_{0}^{\prime}=0, \\
\psi_{i}^{2}=Q_{i} \varphi+\psi_{i+1} \\
2 \psi_{i} \psi_{i}^{\prime}+z^{r} Q_{i}=Q_{i}^{\prime} \varphi+\psi_{i+1}^{\prime}
\end{array}\right\}, \quad i=0,2, \ldots, h-1 .
$$

The entire algorithm consists of the following stages.

Stage 1. Compute the coefficients of the polynomial $\psi(z)$ and of its derivative with respect to $\lambda$ by means of (3.5).

Stage 2. Compute the matrices $U$ and $H$ by means of (3.4).

Stage 3. Compute the matrix $G$, the $s \times s$ right lower submatrix of $\psi^{\prime}(F)$, by using Horner's rule.

Stage 4. Compute the $s \times s$ right lower submatrix $U^{\prime}$ of $\left(F^{n}\right)^{\prime}$ by means of (3.3).

Stage 5. Compute trace $\left(U^{-1} U^{\prime}\right)$.

In view of the structures of the matrices involved, the computational cost of the above algorithm for the evaluation of $p(\lambda) / p^{\prime}(\lambda)$ is roughly twice the cost of the algorithm for the evaluation of $p(\lambda)$, shown in Section 2 . 


\section{The Case of Block Matrices. Let}

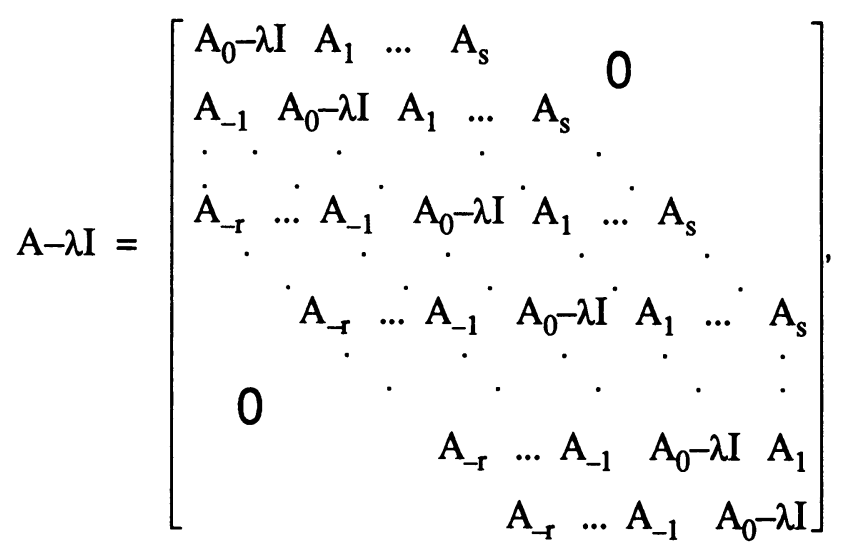

be an $n \times n$ block Toeplitz matrix with $m \times m$ blocks $A_{i}, i=-r, \ldots, s$. We consider the problem of computing $p(\lambda)=\operatorname{det}(A-\lambda I)$ and the ratio $p(\lambda) / p^{\prime}(\lambda)$.

We need to recall some definitions and properties of matrix polynomials (see $[4$, Chapter 7]).

Consider an $m \times m$ matrix $A(z)=\left(a_{i j}(z)\right)$ such that

$$
a_{i j}(z)=\sum_{q=0}^{g} a_{i j}^{(q)} z^{q}
$$

are polynomials in $z$ over $\mathbf{F}$ of degree at most $g$. Then we can write

$$
A(z)=\sum_{q=0}^{g} A_{q} z^{q}
$$

where $A_{q}=\left(a_{i j}^{(q)}\right)$ are $m \times m$ matrices with elements in F, and the matrix $A(z)$ is called an $m \times m$ matrix polynomial.

If $F$ is a given $(m h) \times(m h)$ matrix, then we can define the right value of $A(z)$ at $F$ as the matrix

$$
A(F)=\sum_{q=0}^{g}\left(I_{h} \otimes A_{q}\right) F^{q}
$$

where $I_{h} \otimes B=\operatorname{diag}(B, B, \ldots, B)$ denotes an $h \times h$ block diagonal matrix. For simplicity, throughout the paper we will use the expression

$$
A(F)=\sum_{q=0}^{g} A_{q} F^{q}
$$

to denote the right value $A(F)$.

Given the $m \times m$ matrix polynomials

$$
P(z)=\sum_{i=0}^{p} P_{i} z^{i}, \quad D(z)=\sum_{i=0}^{d} D_{i} z^{i},
$$

such that $D_{d}$ is nonsingular, there exists a unique pair of $m \times m$ matrix polynomials $Q(z)$ and $R(z)$ such that $\operatorname{degree}(R(z))<d$ and

$$
P(z)=Q(z) D(z)+R(z)
$$


$Q(z)$ is said to be the right quotient and $R(z)$ is said to be the right remainder of the division of $P(z)$ by $D(z)$.

We observe that the evaluation of the right quotient and of the right remainder of the division of two matrix polynomials can be performed using known standard algorithms for conventional polynomial division (see [3]), replacing the coefficients with block matrices.

We will use the following two propositions:

Proposition 4.1. Let $Q(z)$ and $R(z)$ be the right quotient and remainder of the division of the $m \times m$ matrix polynomials $P(z)$ by $D(z)$ and let $F$ be a $(p m) \times(p m)$ matrix such that the right value $D(F)$ is the null matrix. Then $P(F)=$ $R(F)$.

Proof. We cannot simply substitute $z=F$ into the matrix equation $P(z)=$ $Q(z) D(z)+R(z)$, for the products of the coefficients of $P(z), Q(z), D(z), R(z)$ with $F$ do not commute. With a bit more caution, however, from the relation

$$
P(z)=Q(z) D(z)+R(z)=\sum_{i=0}^{p-d} Q_{i} D(z) z^{i}+R(z),
$$

where $Q(z)=\sum_{i=0}^{p-d} Q_{i} z^{i}$, we deduce that $P(F)=\sum_{i=0}^{p-d} Q_{i} D(F) F^{i}+R(F)$, whence $P(F)=R(F)$.

PROPOSITION 4.2. Let $Q(z)$ and $R(z)$ be the right quotient and remainder of the division of the matrix polynomials $I z^{p}$ by $D(z)$, respectively, that is,

$$
I z^{p}=Q(z) D(z)+R(z),
$$

and let $S(z), T(z)$ be the right quotient and remainder of the division of $(R(z))^{2}$ by $D(z)$, that is,

$$
(R(z))^{2}=S(z) D(z)+T(z)
$$

Then $T(z)$ is the right remainder of the division of $I z^{2 p}$ by $D(z)$, i.e., there exists a matrix polynomial $U(z)$ such that $I z^{2 p}=U(z) D(z)+T(z)$ (here and hereafter, $I$ denotes the identity matrix of appropriate size).

Proof. From (4.1) we obtain that

$$
(R(z))^{2}=\left(I z^{p}-Q(z) D(z)\right)^{2}=I z^{2 p}+Q(z) D(z) Q(z) D(z)-2 Q(z) D(z) z^{p} .
$$

Therefore, from (4.2) we get

$$
I z^{2 p}=2 Q(z) D(z) z^{p}-Q(z) D(z) Q(z) D(z)+S(z) D(z)+T(z),
$$

whence,

$$
I z^{2 p}=\left(2 Q(z) z^{p}-Q(z) D(z) Q(z)+S(z)\right) D(z)+T(z) .
$$

Similarly to the scalar case, the assumption that $(\mathbf{x}, \lambda)$ is an eigenpair of $A$, that is, $(A-\lambda I) \mathbf{x}=\mathbf{0}, \mathbf{x} \neq \mathbf{0}$, where $\mathbf{x}=\left(\mathbf{x}_{i}\right), \mathbf{x}_{i} \in \mathbf{F}^{m}, i=1,2, \ldots, n$, is a block vector, can be rewritten as the following matrix difference equation of order $k=r+s$ with constant coefficients (see [4, p. 512]),

$$
\begin{aligned}
A_{s} \mathbf{x}_{i+k}+A_{s-1} \mathbf{x}_{i+k-1}+\cdots+\left(A_{0}-\lambda I\right) \mathbf{x}_{r}+\cdots+A_{-r} \mathbf{x}_{i} & =\mathbf{0}, \\
i & =-r,-r+1, \ldots, n-r-1 .
\end{aligned}
$$


This equation is complemented with the following "boundary conditions"

$$
\begin{array}{ll}
\mathbf{x}_{-r}, \ldots, \mathbf{x}_{-1}=\mathbf{0} & \text { (initial conditions) } \\
\mathbf{x}_{n}, \ldots, \mathbf{x}_{n+s-1}=\mathbf{0} & \text { (terminal conditions) }
\end{array}
$$

If $A_{s}$ is nonsingular (the singular case is dealt with in Section 5), the above matrix difference equation is equivalent to

$$
G_{s} \mathbf{x}_{j+k}+G_{s-1} \mathbf{x}_{j+k-1}+\cdots+G_{-r} \mathbf{x}_{j}=\mathbf{0}
$$

where $G_{i}=A_{s}^{-1} A_{i-r}, i=0, \ldots, k, i \neq r, G_{r}=A_{s}^{-1}\left(A_{0}-\lambda I\right)$, so that $G_{k}=I$. Now the matrix difference equation (4.5) with constant coefficients can be rewritten equivalently as follows,

$$
\left[\begin{array}{c}
\mathbf{x}_{i} \\
\vdots \\
\mathbf{x}_{i+k-1}
\end{array}\right]=F^{r+i}\left[\begin{array}{c}
\mathbf{x}_{-r} \\
\vdots \\
\mathbf{x}_{s-1}
\end{array}\right], \quad i=-r,-r+1, \ldots
$$

Here, $F$ denotes the $k \times k$ block companion matrix, compare (2.6), defined by

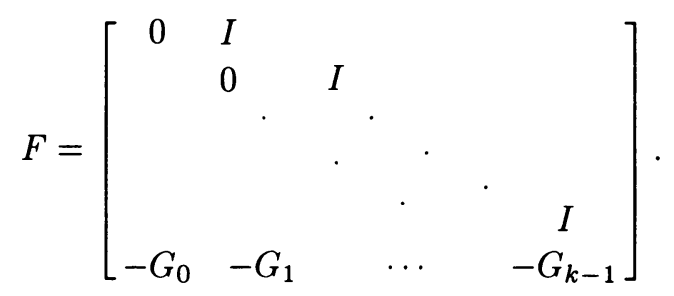

Moreover, from (4.3) and (4.4), we conclude that the equation $\operatorname{det}(A-\lambda I)=0$ is equivalent to $\operatorname{det} U=0$, where $[V \mid U]=\left[0 \mid I_{s m}\right] F^{n}$. The same argument as in the scalar case enables us to prove that $\operatorname{det} U$ is a polynomial in $\lambda$ of degree $n m$ whose term of the highest degree in $\lambda$ is given by

$$
\operatorname{det}\left[\begin{array}{cc}
0 & I_{s-e} \otimes\left(\lambda A_{s}^{-1}\right)^{g} \\
I_{e} \otimes\left(\lambda A_{s}^{-1}\right)^{g+1} & 0
\end{array}\right] .
$$

Here, $g$ and $e$ are natural numbers such that $n=g s+e, e<s$. Therefore, we get the following block version of Proposition 2.1, which has also been proved by Tismenetsky [7], using the spectral theory of matrix polynomials.

PROPOSITION 4.3. Let $U$ be the right lower $s \times s$ block submatrix of the block Frobenius matrix $F^{n}$. Then for the characteristic polynomial $p(\lambda)$ of $A$ we have

$$
p(\lambda)=(-1)^{n s}\left(\operatorname{det} A_{s}\right)^{n} \operatorname{det} U .
$$

Next consider the $m \times m$ matrix polynomial $\Phi(z)=G_{0}+G_{1} z+\cdots+G_{k} z^{k}$ associated with the matrix linear difference equation (4.5). Similarly to the scalar case, we deduce that its right value taken on at $F$ is the null matrix, that is,

$$
\Phi(F)=\sum_{i=0}^{k}\left(I_{m} \otimes G_{i}\right) F^{i}=0 .
$$

Therefore, by virtue of Propositions 4.1 and 4.2 it is possible to compute $F^{n}$ by means of the repeated squaring technique,

$$
\begin{aligned}
& \Psi_{0}=z \\
& \Psi_{i+1}=\Psi_{i} \bmod \Phi(z), \quad i=0,1, \ldots, h-1, h=\log n \\
& F^{n}=\Psi_{h}(F) .
\end{aligned}
$$


Here, the polynomial $\Psi(z) \bmod \Phi(z)$ denotes the right remainder of the right division of $\Psi(z)$ by $\Phi(z)$. Thus, the algorithm for the evaluation of $\operatorname{det}(A-\lambda I)$, described in Section 2, still applies to the case of block matrices in the following form.

Stage 1. Compute the matrices $\Theta_{i}$, the coefficients of the matrix polynomial

$$
\Psi(z)=z^{n} \bmod \Phi(z), \quad \Psi(z)=\sum_{i=0}^{k-1} \Theta_{i} z^{i}
$$

by using the repeated squaring technique

$$
\Psi_{0}=z, \quad \Psi_{i+1}=\Psi_{i}^{2} \bmod \Phi(z), \quad i=0, \ldots, h-1, h=\log _{2} n, \Psi_{h}=\Psi .
$$

Stage 2. Compute the matrix

$$
U=\left[0 \mid I_{s}\right] F^{n}\left[\begin{array}{c}
0 \\
I_{s}
\end{array}\right]=\left[0 \mid I_{s}\right] \Psi(F)\left[\begin{array}{c}
0 \\
I_{s}
\end{array}\right]
$$

by means of the "right" Horner's rule

$$
U=\Theta_{0} L+\left(\Theta_{1} L+\left(\cdots+\left(\Theta_{k-2} L+\Theta_{k-1} L F\right) F\right) \ldots\right) F\left[\begin{array}{c}
0 \\
I_{s}
\end{array}\right],
$$

where $L=\left[0 \mid I_{s}\right]$ is an $s \times k$ matrix; that is, perform the following steps:

$$
\begin{aligned}
Y_{1} & =\Theta_{k-1} L, \\
Y_{i} & =\left(\Theta_{k-i} L+Y_{i-1} F\right), \quad i=2, \ldots, k, \\
U & =Y_{k}\left[\begin{array}{c}
0 \\
I_{s}
\end{array}\right] .
\end{aligned}
$$

Stage 3. Compute $p(\lambda)=(-1)^{n s}\left(\operatorname{det} A_{s}\right)^{n} \operatorname{det} U$.

It is possible to show that the cost of the algorithm is

$$
O\left(\left(m^{2} k \log k+m^{3} k\right) \log (n / k)+m^{3} k^{3}\right)
$$

multiplications if fast polynomial arithmetic is used, and $O\left(m^{3} k^{2} \log (n / k)+m^{3} k^{3}\right)$ multiplications if customary polynomial arithmetic is used.

In a similar way, it is easy to prove that the algorithm for the evaluation of $p(\lambda) / p^{\prime}(\lambda)$, described in Section 3, still applies in the case of block matrices, and its computational cost is roughly doubled with respect to the cost of the evaluation of $p(\lambda)$.

For example, consider the case of the block tridiagonal matrix

$$
A=\left[\begin{array}{cccccccc}
A_{0} & A_{1} & & & & & \\
& & & & & 0 & \\
A_{-1} & A_{0} & & A_{1} & & & & \\
& & & \cdot & \cdot & & & \\
& & . & & \cdot & . & & \\
& 0 & & & & \cdot & . & \\
& & & & A_{-1} & A_{0} & A_{1} \\
& & & & & A_{-1} & A_{0}
\end{array}\right],
$$


and for simplicity, assume $\lambda=0$. Then the bandwidth is $k=2$. Setting $B=$ $A_{1}^{-1} A_{-1}, C=A_{1}^{-1} A_{0}$, we have

$$
\begin{aligned}
& \Phi(x)=I x^{2}+C x+B \\
& \Psi_{1}=x^{2}(\bmod \Phi(x))=-C x-B \\
& \Psi_{i+1}=\Psi_{i}^{2}(\bmod \Phi(x))=C_{i+1} x+B_{i+1}, \quad i=1, \ldots, h-1, h=\log _{2} n .
\end{aligned}
$$

Since

$$
\begin{aligned}
\left(C_{i} x+B_{i}\right)^{2}(\bmod \Phi(x)) & =C_{i}^{2} x^{2}+\left(C_{i} B_{i}+B_{i} C_{i}\right) x+B_{i}^{2}(\bmod \Phi(x)) \\
& =\left(C_{i} B_{i}+B_{i} C_{i}-C_{i}^{2} C\right) x+\left(B_{i}^{2}-C_{i}^{2} B\right),
\end{aligned}
$$

we have the relations

$$
\begin{aligned}
& C_{i+1}=-C_{i}^{2} C+C_{i} B_{i}+B_{i} C_{i}, \\
& B_{i+1}=B_{i}^{2}-C_{i}^{2} B \\
& C_{1}=-C, \quad B_{1}=-B
\end{aligned}
$$

For each $i$ we can compute $C_{i+1}, B_{i+1}$ (given $C, B, C_{i}, B_{i}$ ) in five matrix multiplications, using (4.7) and the equation

$$
C_{i} B_{i}+B_{i} C_{i}=\left(C_{i}+B_{i}\right)^{2}-B_{i}^{2}-C_{i}^{2} .
$$

Now, recall that $F^{n}=\left(I_{m} \otimes C_{h}\right) F+\left(I_{m} \otimes B_{h}\right) I$, so that

$$
U=-C_{h} C+B_{h},
$$

see (4.6).

This means that the overall cost of computing $U$ is not greater than the cost of $5 \log _{2}(n / 2)+1$ matrix multiplications or $m^{3}\left(5 \log _{2}(n / 2)+1\right)$ scalar multiplications.

5. The Degenerate Case. When we evaluated the characteristic polynomial of the block Toeplitz banded matrix in Section 4, we have assumed that the block $A_{s}$ is nonsingular. In that case, it is possible to reduce the original matrix difference equation to the form (4.5) and to solve that equation via powering the companion matrix (4.6). In this section we consider the case where $\operatorname{det} A_{s}=0$.

Suppose we are given the matrix difference equation

$$
A_{s} \mathbf{x}_{j+k}+A_{s-1} \mathbf{x}_{j+k-1}+\cdots+A_{-r} \mathbf{x}_{j}=\mathbf{0}, \quad j=-r, \ldots, n-r-1,
$$

with boundary conditions

$$
\begin{gathered}
\mathbf{x}_{-r}, \ldots, \mathbf{x}_{-1}=\mathbf{0} \\
\mathbf{x}_{n}, \ldots, \mathbf{x}_{n+s-1}=\mathbf{0}
\end{gathered}
$$

where the matrix $A_{s}$ is singular. We may assume, without loss of generality, that

$$
A_{s}=\left[\frac{B_{s}}{0}\right] \text {, }
$$

where $B_{s}$ is a $q \times m$ full-rank matrix, $q<m$; otherwise, consider a nonsingular $m \times m$ matrix $M$ such that

$$
M A_{s}=\left[\frac{B_{s}}{0}\right]
$$


and replace (5.1) with $M A_{s} \mathbf{x}_{j+k}+M A_{s-1} \mathbf{x}_{j+k-1}+\cdots+M A_{-r} \mathbf{x}_{j}=\mathbf{0}, j=$ $-r, \ldots, n-r-1$.

Now partition the matrix $A_{i}$ such that

$$
A_{i}=\left[\frac{B_{i}}{C_{i}}\right]
$$

where $B_{i}$ is a $q \times m$ matrix, so that (5.1) can be split into two equations,

$$
\begin{aligned}
& B_{s} \mathbf{x}_{j+k}+B_{s-1} \mathbf{x}_{j+k-1}+\cdots+B_{-r} \mathbf{x}_{j}=\mathbf{0}, \quad j=-r, \ldots, n-r-1, \\
& C_{s-1} \mathbf{x}_{j+k-1}+\cdots+C_{-r} \mathbf{x}_{j}=\mathbf{0}, \quad j=-r, \ldots, n-r-1 .
\end{aligned}
$$

These equations are equivalent to the following system,

$$
\begin{aligned}
& E_{s} \mathbf{x}_{j+k}+E_{s-1} \mathbf{x}_{j+k-1}+\cdots+E_{-r} \mathbf{x}_{j}=\mathbf{0}, \quad j=-r, \ldots, n-r-2, \\
& C_{s-1} \mathbf{x}_{s-1}+\cdots+C_{-r} \mathbf{x}_{-r}=\mathbf{0}, \\
& B_{s} \mathbf{x}_{n+s-1}+\cdots+B_{-r} \mathbf{x}_{n-r-1}=\mathbf{0},
\end{aligned}
$$

where

$$
E_{i}=\left[\frac{B_{i}}{C_{i-1}}\right], \quad i=-r, \ldots, s, C_{-r-1}=0 .
$$

Assuming $E_{s}$ nonsingular (if $E_{s}$ were singular, we would apply this technique again to the above matrix difference equation), we can express $\mathbf{x}_{i}$ in terms of the block companion matrix

$F=\left[\begin{array}{cccccc}0 & I & & & \\ & 0 & & I & & \\ & & & \cdot & & \\ & & . & & . & \\ & & & \cdot & \cdot \\ -G_{0} & -G_{1} & & \ldots & -G_{k-1}\end{array}\right], \quad G_{i}=-E_{s}^{-1} E_{i-r}, \quad i=0, \ldots, k-1$,

by means of the relations

$$
\left[\begin{array}{c}
\mathbf{x}_{j+1} \\
\cdot \\
\cdot \\
\cdot \\
\mathbf{x}_{j+k}
\end{array}\right]=F^{r+j+1}\left[\begin{array}{c}
\mathbf{x}_{-r} \\
\cdot \\
\cdot \\
\cdot \\
\mathbf{x}_{s-1}
\end{array}\right], \quad j=-r,-r+1, \ldots, n-r-2
$$

$$
\begin{aligned}
& C_{s-1} \mathbf{x}_{s-1}+\cdots+C_{-r} \mathbf{x}_{-r}=\mathbf{0}, \\
& B_{s} \mathbf{x}_{n+s-1}+\cdots+B_{-r} \mathbf{x}_{n-r-1}=\mathbf{0}
\end{aligned}
$$

(5.4) implies that

$$
\left[\begin{array}{c}
\mathbf{x}_{n-r-1} \\
\cdot \\
\cdot \\
\cdot \\
\mathbf{x}_{n+s-r}
\end{array}\right]=F^{n-1}\left[\begin{array}{c}
\mathbf{x}_{-r} \\
\cdot \\
\cdot \\
\cdot \\
\mathbf{x}_{s-1}
\end{array}\right]
$$


Using this last equation and the boundary condition $\mathbf{x}_{n+s-1}=\mathbf{0}$ from (5.3), we may rewrite (5.6) equivalently as follows,

$$
\left[B_{-r} \cdots B_{s}\right] F^{n-1}\left[\begin{array}{c}
\mathbf{x}_{-r} \\
\cdot \\
\cdot \\
\cdot \\
\mathbf{x}_{s-1}
\end{array}\right]=\mathbf{0} .
$$

Then (5.5), (5.6) and the boundary conditions $\mathbf{x}_{n}, \ldots, \mathbf{x}_{n+s-r}=\mathbf{0}$ from (5.3) can be put into the following form,

$$
\left[\begin{array}{ccc}
C_{-r} & \cdots & C_{s-1} \\
W_{-r} & \cdots & W_{s-1} \\
U_{-r} & \cdots & U_{s-1}
\end{array}\right]\left[\begin{array}{c}
\mathbf{x}_{-r} \\
\cdot \\
\cdot \\
\cdot \\
\mathbf{x}_{s-1}
\end{array}\right]=0
$$

where

$$
\begin{aligned}
& {\left[W_{-r} \cdots W_{s-1}\right]=\left[B_{-r} \cdots B_{s-1}\right] F^{n-1}} \\
& {\left[U_{-r} \cdots U_{s-1}\right]=\left[0 \cdots I_{m(s-1)}\right] F^{n-1}}
\end{aligned}
$$

We substitute (5.2) and arrive at the following homogeneous matrix-vector equation with the square coefficient matrix $W$,

$$
\left[\begin{array}{ccc}
C_{0} & \cdots & C_{s-1} \\
W_{0} & \cdots & W_{s-1} \\
U_{0} & \cdots & U_{s-1}
\end{array}\right]\left[\begin{array}{c}
\mathbf{x}_{0} \\
\cdot \\
\cdot \\
\cdot \\
\mathbf{x}_{s-1}
\end{array}\right]=0
$$

This equation has a nontrivial solution if and only if $\operatorname{det} W=0$. Thus we may reduce the solution of the original problem to computing $W$ and $\operatorname{det} W$ for a fixed $\lambda$.

6. A Different Approach Based on Cyclic Reduction. In this section we will examine a different method for computing $\operatorname{det}(A-\lambda I)$ when the matrix $A$ is a block tridiagonal Toeplitz matrix. Observe that this case is quite general, since almost any (block) banded Toeplitz matrix can be considered as a block tridiagonal Toeplitz matrix, with blocks of suitable dimension. This method requires nonsingularity of certain matrices which we need to invert, but it does not require the nonsingularity of the upper diagonal block. Suppose we are given the following $n \times n$ block matrix, where $n=2^{h}-1$,

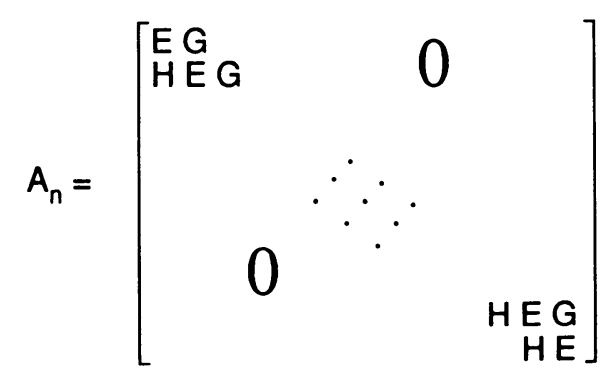


In this case it is possible to apply a cyclic reduction method to compute $\operatorname{det}(A-\lambda I)$. Observe that permuting block rows and block columns of the $n \times n$ block matrix $A-\lambda I$ according to the permutation $\left(1,3,5, \ldots, 2^{h}-1,2,4\right.$, $6, \ldots, 2^{h}-2$ ), yields the matrix

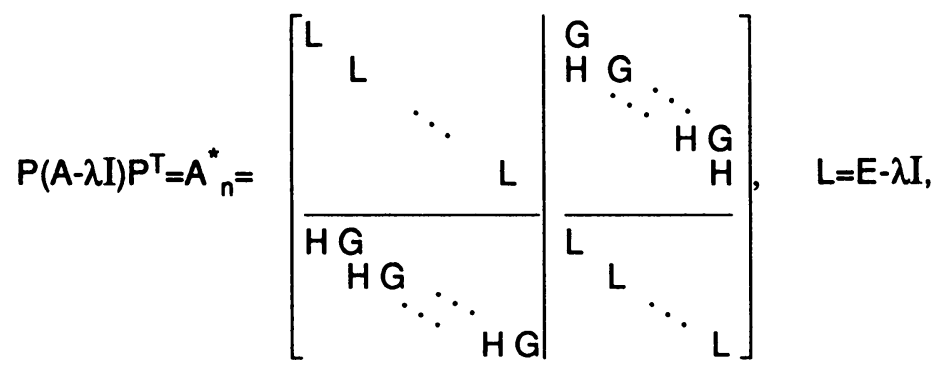

where $P$ is the permutation matrix defining the above permutation.

Moreover, if $\operatorname{det} L \neq 0$, we may perform one step of block Gaussian elimination and write

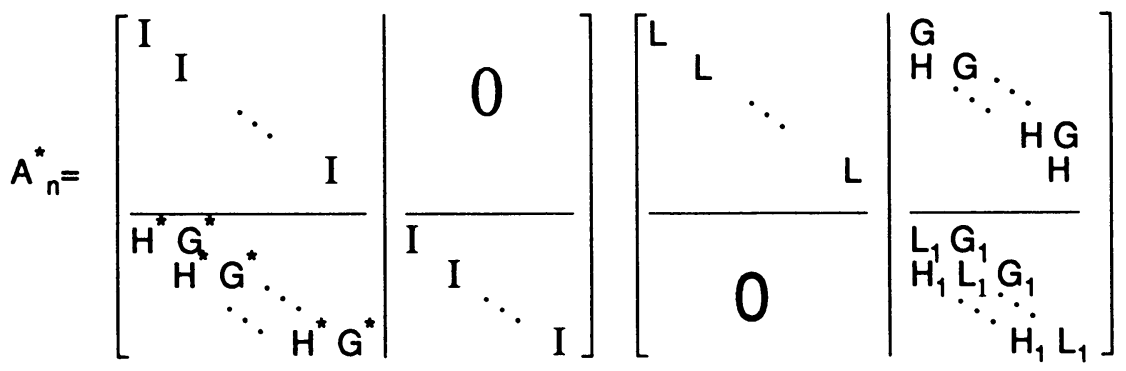

where $H^{*}=H L^{-1}, G^{*}=G L^{-1}$, and

$$
\begin{aligned}
& L_{1}=L-\left(H L^{-1} G+G L^{-1} H\right), \\
& G_{1}=-G L^{-1} G \\
& H_{1}=-H L^{-1} H .
\end{aligned}
$$

Therefore, $\operatorname{det}\left(A_{n}-\lambda I\right)=\operatorname{det} A_{n}^{*}=(\operatorname{det} L)^{(n+1) / 2} \operatorname{det}\left(A_{(n+1) / 2-1}-\lambda I\right)$,

$$
A_{(n+1) / 2-1}=\left[\begin{array}{cccc}
L_{1} & G_{1} & & 0 \\
H_{1} & L_{1} & G_{1} & \\
& 0 & & \\
& & & \\
& & H_{1} L_{1}
\end{array}\right] .
$$

Now, if $L_{1}$ is nonsingular, the same process can be applied to $A_{(n+1) / 2-1}$ and so on. We arrive at the following relations,

$$
\begin{aligned}
\operatorname{det} A_{n}^{*} & =\left(\operatorname{det} L_{0}\right)^{2^{h-1}}\left(\operatorname{det} L_{1}\right)^{2^{h-2}} \cdots\left(\operatorname{det} L_{h-2}\right)^{2} \operatorname{det} L_{h-1}, \\
L_{i+1} & =L_{i}-\left(H_{i} L_{i}^{-1} G_{i}+G_{i} L_{i}^{-1} H_{i}\right) \\
G_{i+1} & =-G_{i} L_{i}^{-1} G_{i} \\
H_{i+1} & =-H_{i} L_{i}^{-1} H_{i} \\
L_{0}=L, \quad H_{0}=H, \quad G_{0}=G, & i=0, \ldots, h-2,
\end{aligned}
$$

provided that all matrices $L_{i}$ are nonsingular. 
The cost of this process is one matrix inversion and six matrix multiplications in each step of the evaluation of $L_{i}, G_{i}, H_{i}$; moreover, the cost of evaluating $h$ determinants should be added. The overall cost is roughly

$$
(22 / 3) m^{3} \log _{2}(n+1)
$$

scalar multiplications.

Observe that the cost of this algorithm is higher than the cost of the algorithm described in Section 5, which required roughly $5 m^{3} \log _{2}(n / 2)$ multiplications. Note, however, that the approach of this section can be applied even when $G$ and $H$ are singular, provided that all $L_{i}$ are nonsingular.

In order to compute the ratio $p(\lambda) / p^{\prime}(\lambda)$ for performing one step of Newton's method, we deduce from (6.1) and (6.2) that

$$
\begin{aligned}
p^{\prime}(\lambda) / p(\lambda)= & 2^{h-1}\left(\operatorname{det} L_{0}\right)^{\prime} /\left(\operatorname{det} L_{0}\right)+2^{h-2}\left(\operatorname{det} L_{1}\right)^{\prime} /\left(\operatorname{det} L_{1}\right) \\
& +\cdots+\left(\operatorname{det} L_{h-1}\right)^{\prime} /\left(\operatorname{det} L_{h-1}\right) .
\end{aligned}
$$

Since $(\operatorname{det} B)^{\prime} /(\operatorname{det} B)=\operatorname{trace}\left(B^{-1} B^{\prime}\right)$, for any nonsingular matrix $B$, it follows that

$$
p^{\prime}(\lambda) / p(\lambda)=2^{h-1} \operatorname{trace}\left(L_{0}^{-1} L_{0}^{\prime}\right)+2^{h-2} \operatorname{trace}\left(L_{1}^{-1} L_{1}^{\prime}\right)+\cdots+\operatorname{trace}\left(L_{h-1}^{-1} L_{h-1}^{\prime}\right) .
$$

The evaluation of the matrices $L_{i}^{\prime}$ can be carried out together with the evaluation of $L_{i}$ by means of the following formulae obtained by taking derivatives of $(6.3)$ with respect to $\lambda$ :

$$
\begin{aligned}
& L_{i+1}^{\prime}=L_{i}^{\prime}-\left(H_{i}^{\prime} L_{i}^{-1} G_{i}+H_{i} L_{i}^{-1} G_{i}^{\prime}+H_{i}\left(L_{i}^{-1}\right)^{\prime} G_{i}\right. \\
& \left.\left.+G_{i}^{\prime} L_{i}^{-1} H_{i}+G_{i} L_{i}^{-1} H_{i}^{\prime}+G_{i}\left(L_{i}^{-1}\right)^{\prime} H_{i}\right)\right\}, \quad i=0, \ldots, h-2 \text {, } \\
& \left.\begin{array}{l}
G_{i+1}^{\prime}=-\left(G_{i}^{\prime} L_{i}^{-1} G_{i}+G_{i} L_{i}^{-1} G_{i}^{\prime}+G_{i}\left(L_{i}^{-1}\right)^{\prime} G_{i}\right) \\
H_{i+1}^{\prime}=-\left(H_{i}^{\prime} L_{i}^{-1} H_{i}+H_{i} L_{i}^{-1} H_{i}^{\prime}+H_{i}\left(L_{i}^{-1}\right)^{\prime} H_{i}\right)
\end{array}\right\} \\
& L_{0}^{\prime}=I, \quad G_{0}^{\prime}=0, \quad H_{0}^{\prime}=0 .
\end{aligned}
$$

Here $\left(L_{i}^{-1}\right)^{\prime}$ can be computed using the formula

$$
\left(L_{i}^{-1}\right)^{\prime}=-L_{i}^{-1}\left(L_{i}\right)^{\prime} L_{i}^{-1} .
$$

Dipartimento di Matematica

II Università di Roma "Tor Vergata"

Rome, Italy

Department of Computer Science

SUNY at Albany

Albany, New York 12222

1. D. BINI \& M. CA POVANI, "Fast parallel and sequential computations and spectral properties concerning band Toeplitz matrices," Calcolo, v. 22, 1983, pp. 176-189.

2. D. BINI \& M. CAPOVANI, "Spectral and computational properties of band symmetric Toeplitz matrices," Linear Algebra Appl., v. 52/53, 1983, pp. 99-126.

3. D. BINI \& V. PAN, "Polynomial division and its computational complexity," J. Complexity, v. 2, 1986, pp. 179-203.

4. P. Lancaster \& M. Tismenetsky, The Theory of Matrices, Academic Press, New York, 1985. 
5. V. PAN, On the Sequential and Parallel Approximate Evaluation of Polynomial Zeros, Tech. Rep. 87-14, Computer Science Dept., SUNYA, Albany, N. Y., 1987, to appear in Comput. Math. Appl.

6. J. StOER \& R. BUlirSCH, Introduction to Numerical Analysis, Springer-Verlag, Berlin, 1980.

7. M. TISMEnEtSKy, "Determinant of block-Toeplitz band matrices," Linear Algebra Appl., v. 85, 1987, pp. 165-187.

8. W. F. TRENCH, "On the eigenvalue problem for Toeplitz band Matrices," Linear Algebra Appl., v. 64, 1985, pp. 199-214. 